\title{
On the semiannual and annual variations of geomagnetic activity and components
}

\author{
J.-L. Le Mouël ${ }^{1}$, E. Blanter ${ }^{2}$, A. Chulliat ${ }^{1}$, and M. Shnirman ${ }^{2}$ \\ ${ }^{1}$ Laboratoire de Géomagnétisme, Institut de Physique du Globe de Paris, 4 place Jussieu, 75252 Paris cedex 05, France \\ ${ }^{2}$ International Institute of Earthquake Prediction Theory and Mathematical Geophysics, Warshavskoye sh. 79 kor. 2, \\ Moscow 113556, Russia
}

Received: 9 January 2004 - Revised: 18 May 2004 - Accepted: 2 June 2004 - Published: 3 November 2004

\begin{abstract}
The semiannual and annual lines in a long series of magnetic observatories daily values, as well as in the $a a$-activity index series, are investigated. For both periods, amplitudes and phases of the lines corresponding to the different series present grossly common variations on decadal time scales; relative phases and amplitude ratios between the observatories change with the same time constants. The results are briefly discussed with regards to commonly received theories of the semiannual variation of magnetic activity, and some possible mechanisms for the observed geographical variability are suggested.
\end{abstract}

Key words. Geomagnetism and Paleomagnetism (Time variations, diurnal to secular) - Magnetospheric physics (current systems; solar wind-magnetosphere interactions)

\section{Introduction}

"One of the earliest recognized patterns in geomagnetic activity was its semiannual variation (Cortie, 1912; Chapman and Bartels, 1940). The various explanations for this variation, proposed over the years, can be divided roughly into two classes: the axial hypothesis, in which the heliographic latitude of the Earth plays a role, and the equinoctial hypothesis in which the orientation of the Earth's axis of rotation relative to the Earth-Sun line plays a role." We quoted here the first lines of the paper by Russell and McPherron (1973). In an attempt to reconcile both hypothesis, these authors proposed a new model wherein the semiannual variation is due to the variation of the angle between the cross-flow component of the solar-wind magnetic field and that of the Earth's magnetic dipole axis. This model predicts peaks on 5 April and on 5 October, whereas previous models predicted peaks either on 5 March and on 5 September (axial hypothesis), or on 21 March and on 21 September (equinoctial hypothesis).

The Russell and McPherron model agrees the best with the observations and therefore is now widely accepted. Yet very

Correspondence to: A. Chulliat

(chulliat@ipgp.jussieu.fr) little is known about the long-term variation, not only of the phase but also of the amplitude of the semiannual variation. These variations do exist: for example, in their analysis of the $U$ index series from 1872 to 1930 , Russell and McPherron (1973) note that "the dates of the maximums and minimums" are "surprisingly variable" between quiet and disturbed years.

In a recent paper (Le Mouël et al., 2004), we focussed on the time variation of the amplitude of the semiannual variation. The data included $X$ - (northward) and $Y$-(eastward) components of the magnetic field as recorded in 9 observatories around the world since the beginning of the 20th century, as well as the $a a$-index series (Mayaud, 1972). Figure 1 gives an illustration of the semiannual variation of the $a a$-index. We showed that the amplitude of the 6-month line for $X$ and $Y$ presents a smooth, large oscillation over the last 70 years, with a pseudo-period of some 50 years and a relative variation reaching $50 \%$ at some observatories. The amplitude of the 6-month line for the $a a$-index was found to follow a very similar trend up to 1980 and to diverge after that date. However, this divergence was due to an error in our data set after 1980.

In the present paper we will focus on the phase of the semiannual variation and investigate its time variation. Long series of both the (correct) $a a$-index and the $X$-component of the magnetic field will be considered. We will also present, more briefly, the results of a study of the amplitude and phase of the annual variation of the $X$-component and $a a$-index.

\section{Data and analysis}

The $X$-component data analysed in the present paper are daily mean values from 9 observatories around the world. Name, location and years available for each observatory are given in Table 1. Observatories were selected according to the length and quality of the series and to their geographic location. Daily mean values were obtained by Bellanger et al. (2002) from hourly means provided by the World Data Center in Copenhagen, Danemark (ftp://ftp.dmi.dk/pub/wdcc1), or directly by the observatories. 
Table 1. Names, codes and coordinates of magnetic observatories used in the study, and years available.

\begin{tabular}{llcccrc}
\hline \multicolumn{1}{c}{ Name } & Code & $\begin{array}{c}\text { Geographical } \\
\text { latitude }\end{array}$ & $\begin{array}{c}\text { Geographical } \\
\text { longitude }\end{array}$ & $\begin{array}{c}\text { Geomagnetic } \\
\text { latitude }\end{array}$ & $\begin{array}{c}\text { Geomagnetic } \\
\text { longitude }\end{array}$ & $\begin{array}{c}\text { Years } \\
\text { available }\end{array}$ \\
\hline Chambon-la-Forêt & CLF & 48.02 & 2.26 & 43.36 & 79.32 & $1944-2000$ \\
Eskdalemuir & ESK & 55.30 & 356.80 & 52.15 & 76.30 & $1914-2000$ \\
Hartland & HAD & 50.99 & 355.22 & 47.53 & 74.74 & $1926-2000$ \\
Hermanus & HER & -34.42 & 19.23 & -42.43 & 82.62 & $1940-2000$ \\
Honolulu & HON & 21.32 & 202.00 & 21.39 & 269.92 & $1904-1976$ \\
Kakioka & KAK & 36.23 & 140.19 & 29.33 & 211.88 & $1925-2000$ \\
Lerwick & LER & 60.05 & 358.82 & 57.86 & 80.95 & $1926-2000$ \\
Sitka & SIT & 57.07 & 224.67 & 59.71 & 280.30 & $1940-2000$ \\
Tucson & TUC & 32.25 & 249.17 & 39.80 & 314.48 & $1914-1968$ \\
\hline
\end{tabular}

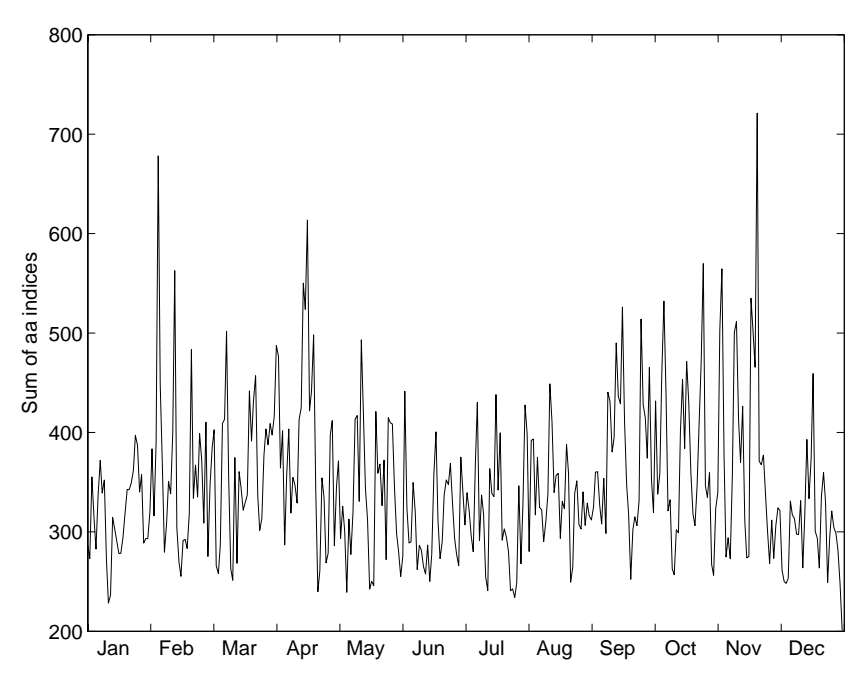

Fig. 1. Annual variation of the $a a$-index, summed over 22 years from 1868 to 1890 .

We will not recall the definition of the magnetic activity three hourly index $a a$ (see Mayaud, 1972, 1980), whose series goes back to 1868 . The $a a$-index characterizes the geomagnetic activity; it has been recently used to reconstruct the solar irradiance before 1975, i.e. at dates where direct measurements are not available (Lockwood et al., 1999; Solanki et al., 2002). Here we consider daily averages of $a a$ (i.e., for a given day $a a$ is the average of the 8 indices of the day) provided by the National Geophysical Data Center (ftp://ftp.ngdc.noaa.gov). Note that the $a a$-index is mostly derived from the $X$-component (though sometimes from the $Y$-component) and that HAD is one of the current $a a$-index observatories (from 1957).

Another time series used in this paper is that of the sunspot number, which goes back to the 17 th century; we used only data from the last century.

The $X$-component and $a a$-index are analysed using the same method as in Le Mouël et al. (2004). First, a running mean over 31 days is computed backward for each day of the series; this eliminates high frequency variations. (The phase change introduced by this running mean is corrected at the end of the analysis.) Second, a band-pass linear filter centered on a period of about 5 months is applied to the series, filtering out variations of periods larger than 2 years and smaller than 2 months (see the full description of the filter in Le Mouël et al., 2004). Third, the 6-month and 12-month Fourier coefficients are computed on a sliding time window of length 22 years (i.e. in length two solar cycles):

$$
\begin{aligned}
& \mathcal{A}_{N}(k)+i \mathcal{B}_{N}(k)= \\
& \frac{1}{2 \tau+1} \sum_{\rho=k-\tau}^{k+\tau} \eta(\rho)\left(\cos \frac{2 \pi}{T_{N}} \rho+i \sin \frac{2 \pi}{T_{N}} \rho\right),
\end{aligned}
$$

where $N=6$ or $12, k$ is the day, $2 \tau+1=8035$ (number of days in 22 years), $\eta(\rho)$ is the value at day $\rho$ of the filtered time series (obtained at the second step) and $T_{N}=182.62$ days or 365.24 days. The amplitude of this line is

$$
A_{N}(k)=\left(\mathcal{A}_{N}^{2}(k)+\mathcal{B}_{N}^{2}(k)\right)^{1 / 2}
$$

and its phase is

$$
\Phi_{N}(k)=\tan ^{-1} \frac{\mathcal{B}_{N}(k)}{\mathcal{A}_{N}(k)} .
$$

Note that the length of the window must be a large enough multiple of one year (in order to detect the semiannual and annual lines) and a multiple of eleven years (in order to average out the effects of the solar cycle), but not to exceed a few decades (in order not to smooth too much the decadal variations we are looking for). This is the reason why we chose a sliding window 22 years in length.

\section{Amplitude and phase of the semiannual variation}

\section{$3.1 a a$-index}

The amplitude of the semiannual variation of the $a a$-index is plotted in Fig. 2. The amplitude plot before 1980 is identical to that published in Le Mouël et al. (2004); after that date it has been corrected. 


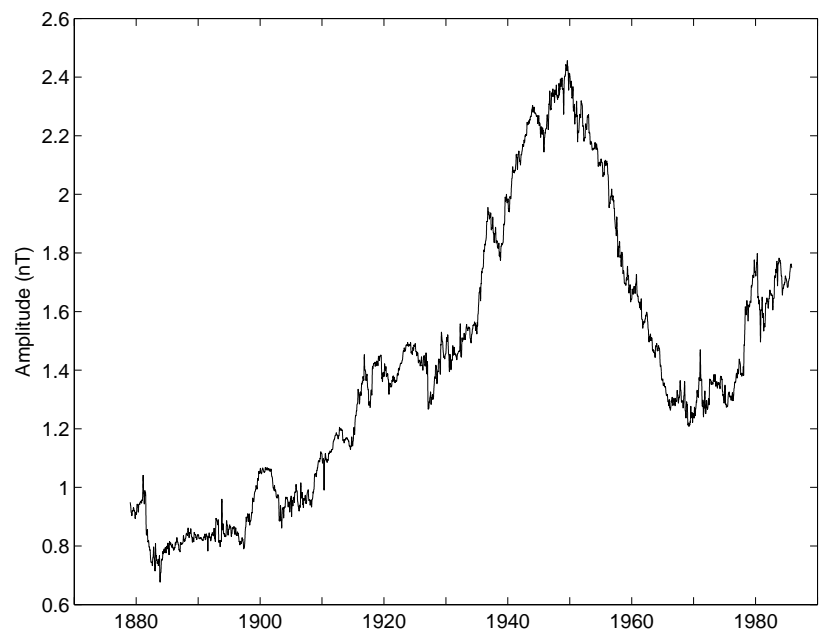

Fig. 2. Time variation of the amplitude of the $a a$-index semiannual variation, computed using a 22 -year sliding window, centered.

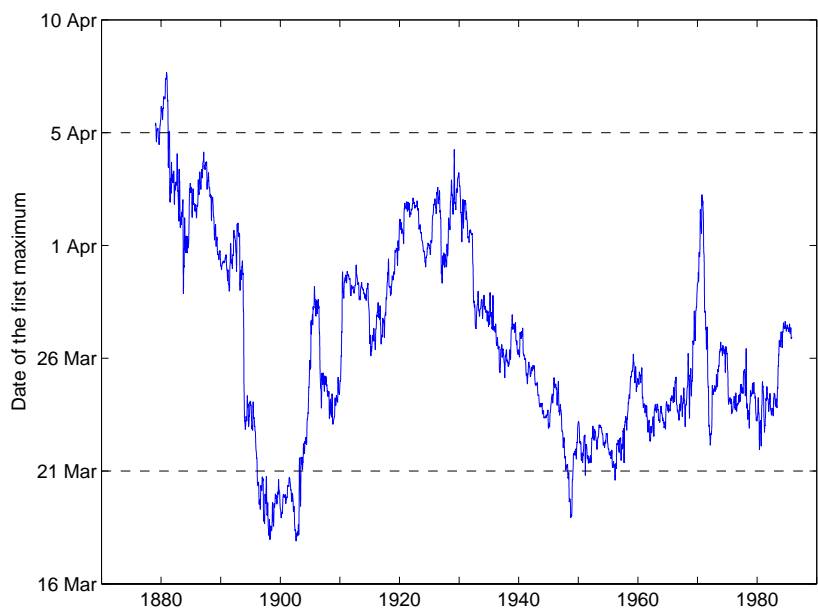

Fig. 3. Time variation of the phase of the $a a$-index semiannual variation, computed using a 22 -year sliding window, centered. The phase is reckoned as the date of the first maximum.

The phase, reckoned as the date of the first maximum of the year, is plotted in Fig. 3. The 21 March would be the date of the maximum for a purely equinoctial mechanism; 5 April is the date of the maximum predicted by the Russell and McPherron model. The observed phase seems to ocillate between these two dates: counting time backwards, the maximum occurs, on average, two days after 21 March from 2000 to 1950 , two days before 5 April from 1930 to 1920, two days before 21 March around 1900 and on 5 April around 1880. (Let us stress again that the results of the analysis of a 22-year interval are referred to the center of the interval.) Also a large peak appears around 1970. Note that the amplitude of this oscillation, about 20 days, is by no means a small time shift compared to 90 days, the half period of the studied variation.

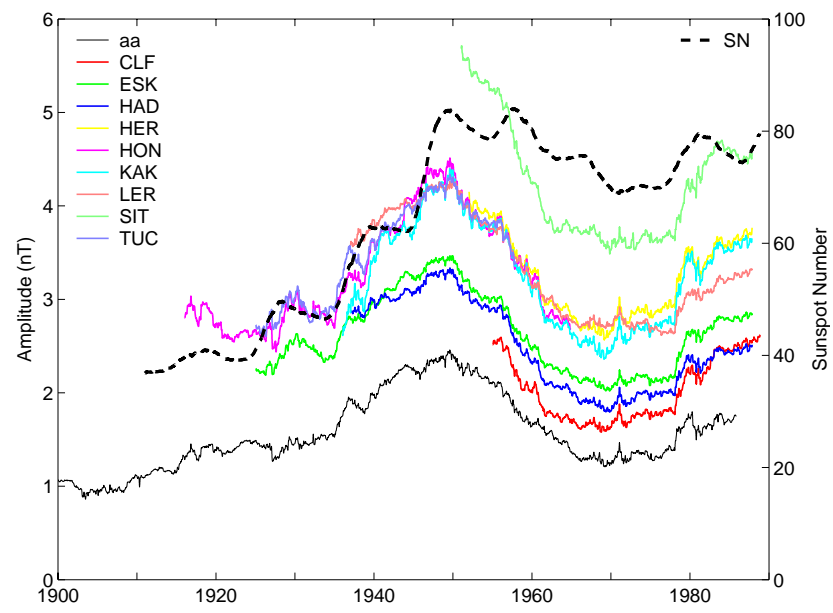

Fig. 4. Time variation of the amplitude of the semiannual variation of the $X$-component in nine observatories (left ordinate axis), computed using a 22-year sliding window, centered. The sunspot number (right ordinate axis) and the amplitude of the semiannual variation of the $a a$-index (left ordinate axis), computed using the same window, are also represented.

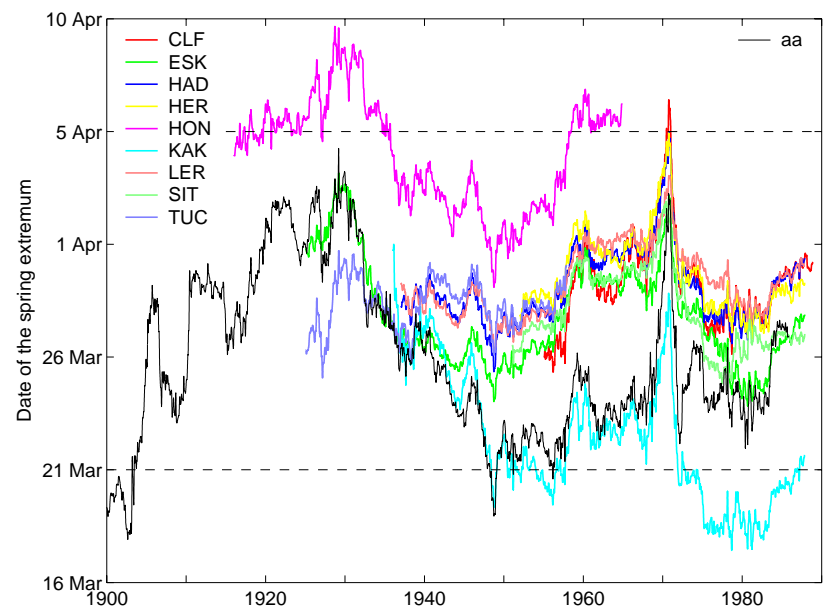

Fig. 5. Time variation of the phase of the semiannual variation of the $X$-component in nine observatories, computed using a 22-year sliding window, centered. The phase of the $a a$-index semiannual variation, computed using the same window, is also represented. Phases are reckoned as the dates of the spring extrema.

\section{$3.2 X$-component}

Figures 4 and 5 show the time variation of $A_{6}(k)$ and $\Phi_{6}(k)$ at the various observatories. (Remember that there is an estimate $A_{6}(k)$ and $\Phi_{6}(k)$ per day and that the length of the series depends on the observatory.) Amplitude and phase of the $a a$ semiannual variation have been added to the figure for easier comparison, as well as the sunspot number.

It is remarkable that the amplitudes at all the observatories vary the same way and the same way as $a a$. This result was already obtained in Le Mouël et al. (2004). 


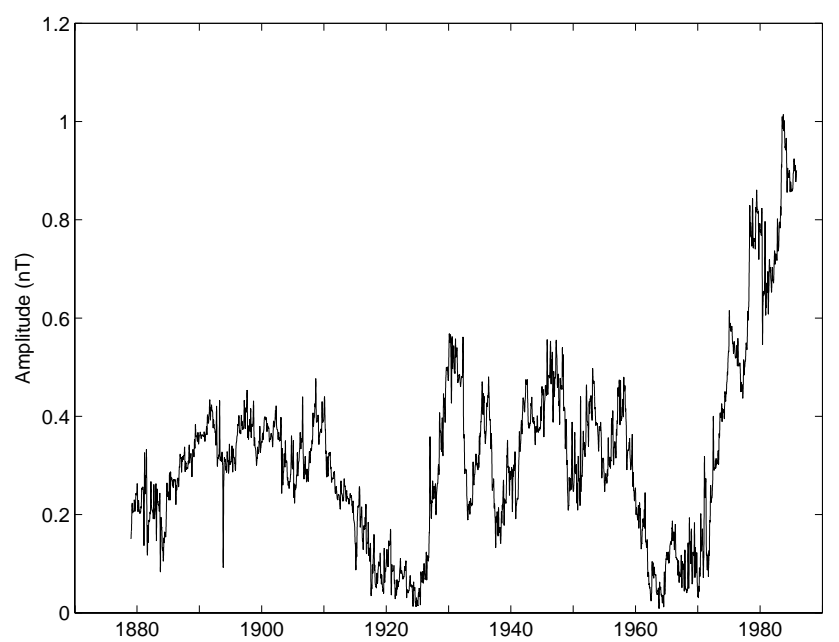

Fig. 6. Time variation of the amplitude of the $a a$-index annual variation, computed using a 22-year sliding window, centered.

Let us now look at the phases, reckoned as the dates of the spring extrema. It appears that:

1. The phases vary grossly the same way at all observatories, and the same way as the phase of the $a a$-index on the common time span.

2. The phase variations are of the order of ten days and are comprised between a few days before 21 March and a few days after 5 April, as already observed for $a a$; however, the oscillatory character is somewhat less pronounced in the observatories.

3. There are significant phase differences between some of the observatories. For example, from 1991 to 1950 the phase difference between KAK and HON, approximately constant, is more than 12 days.

4. Phase differences between observatories vary in time. Apart from HON, all the observatories have phases close to one another around 1930, whereas later they could differ by a week.

5. The phase at one observatory, KAK, is strikingly similar to that of $a a$ from 1935 to 1970.

6. A sharp and intense peak appears on all phase curves around 1970, to which corresponds a small but clear peak on all the amplitudes.

\section{Amplitude and phase of the annual variation}

\section{$4.1 a a$-index}

Figures 6 and 7 display the amplitude and phase of the $a a$ index annual variation. Since $a a$ is computed from two antipodal observatories (and adequate corrections applied; see Mayaud, 1972, 1980), a large annual variation is unexpected.

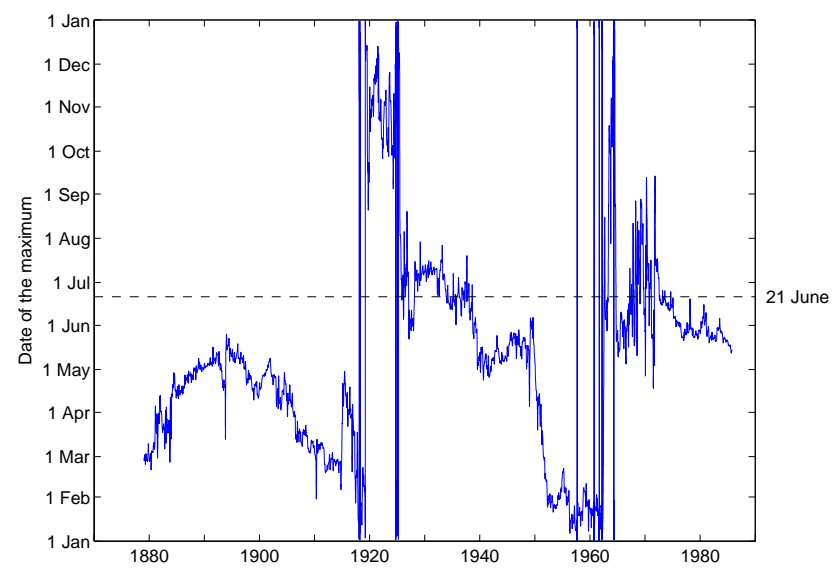

Fig. 7. Time variation of the phase of the $a a$-index annual variation, computed using a 22-year sliding window, centered. The phase is reckoned as the date of the maximum.

Amplitude is indeed weak compared to that of the semiannual variation (see Fig. 2) or that observed at the observatories (see Fig. 8), except in recent years, where it has increased up to 140 in 1990 . These values might be thought to be unsignificant and represent some noise. Nevertheless, the amplitude and phase patterns are rather well organized and present clear similarities. Phase is close to its expected value, 21 June, except before 1920, where it is always earlier than June, and around 1920 and 1960. At these epochs the amplitude is too weak to allow stable estimates of the phase; hence the one-year high vertical bars. Therefore, we believe the general patterns of Figs. 6 and 7 to be real.

\section{$4.2 X$-component}

The amplitude $A_{12}$ and phase $\Phi_{12}$ of the $X$-component at the various observatories are represented in Figs. 8 and 9. Although they resemble each other, the amplitude curves do not look as similar to each other as in the case of the semiannual variation. Large variations in amplitude occur; for example, the amplitude of KAK varies from 50 to 300 ! There are also large relative variations; for example, the ratio of amplitudes at KAK and LER varies from 1.1 to 0.2 in three decades.

Phase curves definitely share a common trend, except for the beginning of the ESK series. From 1990 to 1970 most of them follow each other inside a two-week band around the expected time of summer extremum, 21 June. Going backward in time, phases at KAK and HON take high positive (ahead of 21 June) values, up to, respectively, 40 days and 20 days. The phase variation at HON reaches 2 months. The difference in phase between LER and KAK (or HON) goes from zero to 1 month. Note that the estimation of the phase at KAK is unstable before 1940 due to the very low amplitude. 


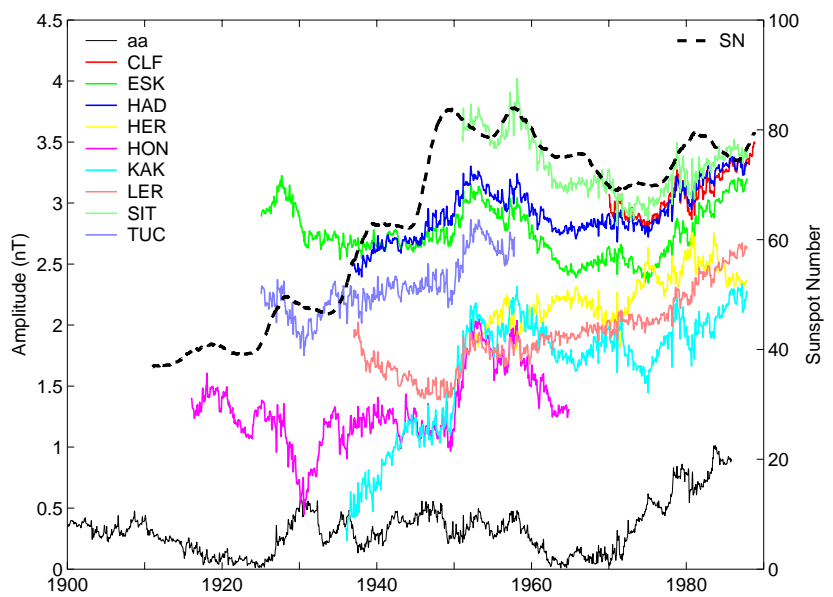

Fig. 8. Time variation of the amplitude of the annual variation of the $X$ component in nine observatories (left ordinate axis), computed using a 22-year sliding window, centered. The sunspot number (right ordinate axis) and the amplitude of the annual variation of the $a a$-index (left ordinate axis), computed using the same window, are also represented.

\section{Discussion}

The first striking result is the large change in the aa 6-month line amplitude, already pointed out in Le Mouël et al. (2004), but now corrected and extended backwards in time. The curve of Fig. 2 has the characteristic shape of the curves representing the time averaged variation of various magnetic parameters (Le Mouël et al., 2004) and the sunspot number, but with an amplification of the range. If the change in sunspot number is attributed to a change in solar activity (which is close to a truism), it can be said that the amplitude of the $a a$ semiannual line does not react linearly to this change.

The date of the first maximum (Fig. 3) is alternately close to the value expected from a purely equinoctial mechanism, 21 March, and to that predicted by the Russell and McPherron model, 5 April, using a value of $11^{\circ}$ for the tilt of the Earth's magnetic dipole axis. There seems to be no trace of a purely axial effect. This result suggests that some part of the mechanism controlling the semiannual variation is not explained by the Russell and McPherron model.

The amplitude and phase of the semiannual line of the $X$ component, in all observatories, closely follow the $a a$ one, even often in fine details (Figs. 4 and 5). One can safely conclude that the ionospheric and magnetospheric currents' system responsible for the $a a$ and field components is the same. That is by no means trivial: $a a$ is a three-hourly activity index, whereas the $X$ series is composed of the daily average values of this component. In the first case we have a semiannual modulation of some measure of short-term components of the field components, in the second case a genuine long-term (6 months) variation of these components.

The amplitude of the annual line varies with the observatory (Fig. 8), which is not a surprise (although the geographical distribution of this amplitude is poorly understood). But

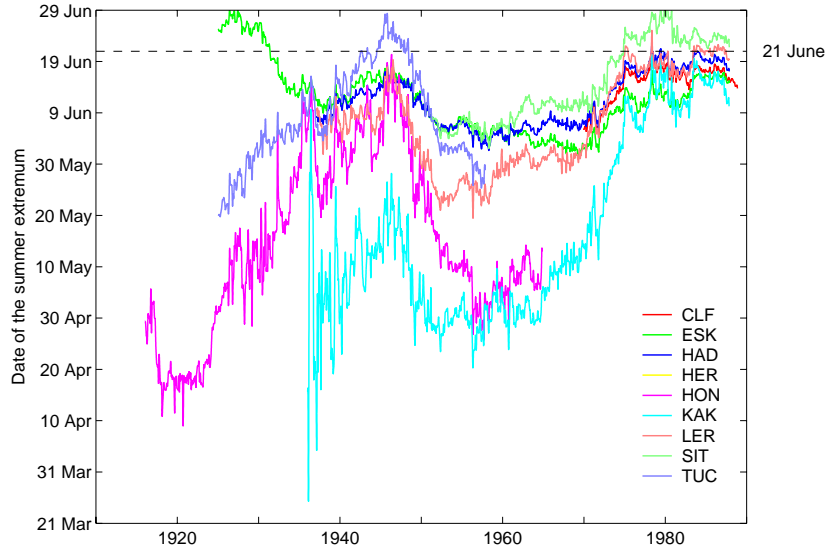

Fig. 9. Time variation of the phase of the annual variation of the $X$ component in nine observatories, computed using a 22-year sliding window, centered. Phases are reckoned as the dates of the summer extrema.

the phase varies too (Fig. 9), from an observatory to the other, with differences reaching a month. This is less expected and reflects some rather complex generation mechanism. All the more, since the phase varies on the considered time span in all observatories, as the amplitudes do (so phase differences between observatories cannot be interpreted simply as induction effects, for example, induction in the oceans). More intriguing, the relative phases (i.e. the phase differences between the observatories) evolve with time.

The variation of the solar activity responsible for the mean variation of the lines' amplitudes cannot produce directly the time variations of the relative phases and amplitude ratios (between the different observatories). The responsible direct mechanism is linked to the Earth: the ionospheric and magnetospheric current systems generating the annual and semiannual lines (whatever they are) change their geometry and characteristics on the observed decadal time scales. How can this be?

1. These geometry changes could be due to the time changes in the solar activity and solar wind themselves, through complex MHD interactions. That would explain why relative changes seem to have the same time constants as the average trend in Figs. 3 and 4.

2. They could be due to changes in the internal magnetic field. The secular variation (SV) of the main field is not (despite its name) that slow and regular. It contains decadal time scales (e.g. Hulot and Le Mouël, 1994); and non-dipolar components of the field may change by their order of magnitude in some 200 years; in some places the field changes by more than $100 \mathrm{nT} / \mathrm{yr}$ (i.e. $10 \%$ on some 20 years). Note that the peaks in the phases of the semiannual variation in 1970 (Figs. 4 and 5) correspond to a brutal acceleration of the north magnetic pole velocity associated with the 1969 geomagnetic jerk (Mandea and Dormy, 2003). 
3. Less plausible, some coupling with a dense lower atmosphere could be invoked. Note that the rapid and accelerated increase in the $a a$-index annual line amplitude in the last decades (Fig. 6) is somewhat analogous to that of the global temperature (Solanki, 2002).

Remark: When dealing with the annual variation of the field components, one may be concerned by the possibility of a spurious temperature effect on the ancient magnetometers (the observation that phases are less scattered in recent years might buttress this view). However, we are confident in the data for the following reasons: a) the temperature effect used is to be taken into account by regular absolute measurements in good observatories, among which the listed ones; $b$ ) the temperature effect is more critical for the vertical component recording (the magnetic balance was equipped with a big magnet). $X$ variometer was much easier to manage; c) most of all, the resemblance between the curves (amplitude and phase) from the different observatories makes this hypothesis untenable (in the case of the semiannual line we have the definitive argument of the close similarity with $a a$; such a clue cannot be expected in the case of the 12-month lines as discussed below).

The existence of the $a a$ annual line shows that the balance between the two antipodal observatories is not perfect, which is not surprising. And the time variation of its amplitude over 120 years (Fig. 6) indicates that the balance evolves, which is quite expected from the above discussion of the time changes of the various amplitudes and phases.

\section{Conclusions}

In the present paper we have analysed the long-term variations of the amplitude and phase of the 6-month and 12-month lines in the spectrum of the $a a$-index and $X$ component at various observatories. These variations are complicated and sometimes difficult to interpret, but three results stand out.

The amplitude of the 6 months line varies in the same way at all considered observatories and in the $a a$-index. This variation appears correlated with that of the sunspot number, which confirms that it originates in variations of the solar activity. (Note that a similar trend has been observed in global temperature by Solanki, 2002.)

The phase of the 6-month line, in the $X$-component at various observatories as in the $a a$-index, oscillates between 21 March and 5 April, i.e. between the date predicted by a purely equinoctial mechanism and the model by Russell and McPherron (1973). This variation does not seem to be correlated with solar activity. Significant time variations of the relative phases between observatories are also observed. This phenomenon could be caused by variations in non-dipolar components of the Earth's internal magnetic field; the sudden phase shift occuring in 1970 might then be explained by a sudden acceleration of the variations of these components.

The phase of the 12-month line varies in time in a coherent manner at all considered observatories, but at the same time the relative phases between various observatories vary significantly. Here again, this observation could be explained by a modification of the ionospheric and magnetospheric current systems due to variations in the non-dipolar components of the internal magnetic field.

Whatever the value of these tentative interpretations, we think these observations are worth attention.

Acknowledgements. This is IPGP contribution No. 2911. We thank two anonymous referees for helping in significantly improving the manuscript.

Topical Editor T. Pulkkinen thanks J. Strestik and another referee for their help in evaluating this paper.

\section{References}

Bellanger, E., Blanter, E. M., Le Mouël, J.-L., Mandea, M., and Shnirman, M. G.: On the geometry of the external geomagnetic irregular variations, J. Geophys. Res., 107, 1414, 2002.

Chapman, S. and Bartels, J.: Geomagnetism, Oxford University Press, 1940.

Cortie, A. L.: Sunspots and terrestrial magnetic phenomena, 18981911, Mon. Notic. Roy. Astron. Soc., 73, 52-60, 1912.

Hulot, G. and Le Mouël, J.-L.: A statistical approach to the Earth's main magnetic field, Phys. Earth Planet. Inter., 82, 167-183, 1994.

Le Mouël, J.-L., Blanter, E., and Shnirman, M.: The six-month line in geomagnetic long series, Ann. Geophys., 22, 985-992, 2004.

Le Mouël, J.-L., Kossobokov, V., and Courtillot, V.: On long-term variations of simple geomagnetic indices and slow changes in magnetospheric currents; the emergence of global warming after 1990?, Earth Planet. Sci. Lett., in press, 2004.

Lockwood, M., Stamper, R., and Wild, M. N.: A doubling of the Sun's coronal magnetic field during the past 110 years, Nature, 399, 437-439, 1999.

Mandea, M. and Dormy, E.: Asymmetric behaviour of magnetic dip poles, Earth Planets Space, 55, 153-157, 2003.

Mayaud, P. N.: The $a a$ indices: A 100-year series characterizing the magnetic activity, J. Geophys. Res., 77, 6870, 1972.

Mayaud, P. N.: Derivation, Meaning and Use of Geomagnetic Indices, Geophysical Monograph 22, AGU, Washington, D.C., 1980.

Russell, C. T. and McPherron, R. L.: Semiannual variation of geomagnetic activity, J. Geophys. Res., 78, 92-108, 1973.

Solanki, S. K.: Solar variability and climate change: is there a link?, Astron. Geophys., 43, 5.9-5.13, 2002.

Solanki, S. K., Schüssler, M., and Fligger, M.: Secular variation of the Sun's magnetic flux, Astron. Astrophys., 383, 706-712, 2002. 